\title{
Evaluación de dos sistemas de tutorado para el cultivo de la arveja voluble (Pisum sativum L.) en condiciones de la Sabana de Bogotá
}

\section{Evaluation of two training systems for indeterminate pea (Pisum sativum L.) culture in conditions of the Bogota Plateau}
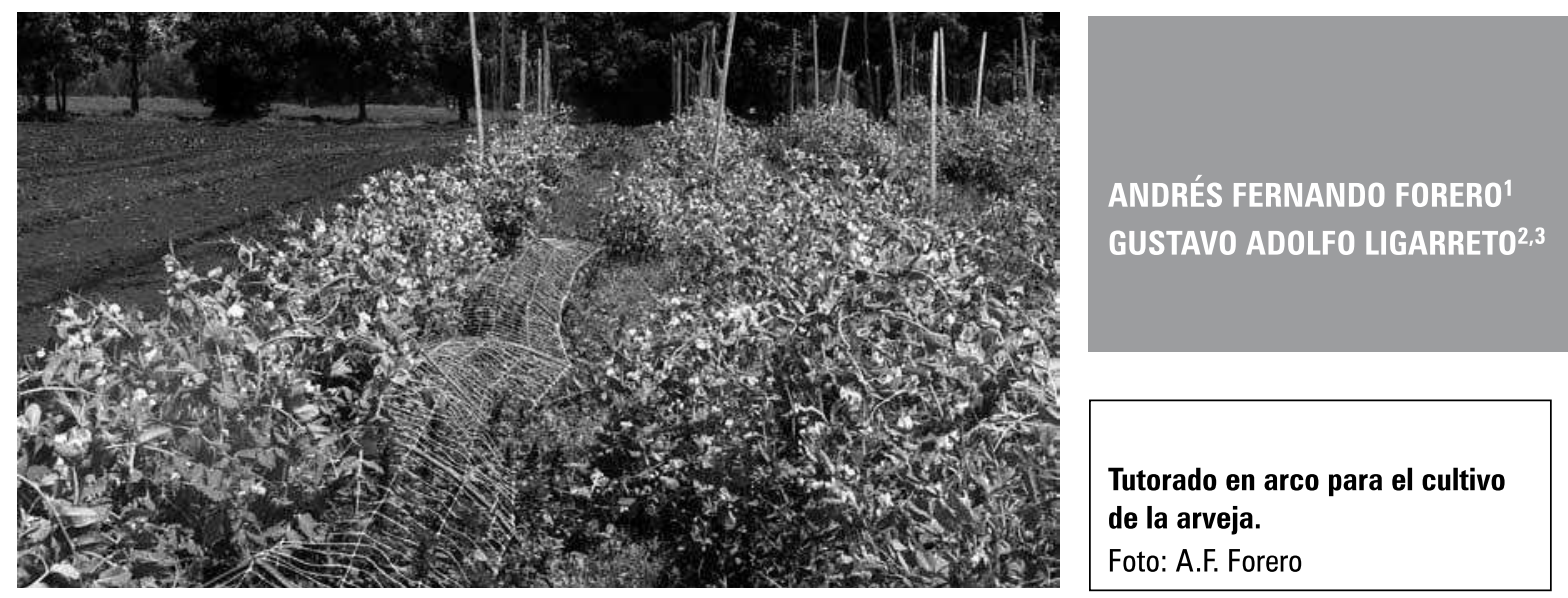

\section{RESUMEN}

Se evaluaron dos sistemas de tutorado; el tutorado vertical y el de arco, que consiste en una propuesta de innovación de los sistemas tradicionales, en el que se recurrió a nuevos materiales y disposiciones que no afecten las densidades siembra que se manejan en la actualidad y permitan el ingreso de maquinaria para el desarrollo de labores durante el ciclo del cultivo. El ensayo comprendió dos partes: la determinación de la estructura adecuada para el tutorado en arco y la evaluación de la viabilidad técnica y económica de este sistema de cultivo de la arveja voluble y su comparación con el tutorado tradicional. Para la estructura del tutorado en arco se utilizó guadua, fibra de polipropileno y mallas y se evaluaron: distancia entre guaduas, enganche entre guaduas y el tamaño del hueco interno de la malla. Los resultados en la primera parte del ensayo permitieron definir que el sistema de tutorado en arco debe tener una distancia entre guaduas de dos metros, un solo enganche entre guaduas y una malla de $15 \times 17 \mathrm{~cm}^{2}$ de huecos interiores. En la segunda parte del ensayo, el tutorado en arco presentó un rendimiento menor que el tutorado vertical, costos de instalación más altos y una vida útil más larga.

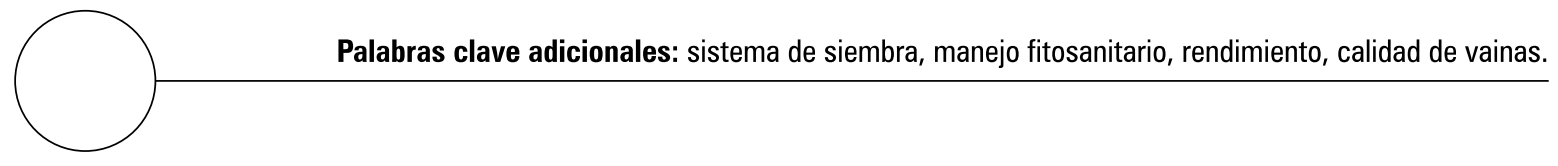

\footnotetext{
1 Programa Agricultura Sostenible, Centro de Investigaciones y Asesorías Agroindustriales (CIAA), Universidad de Bogotá Jorge Tadeo Lozano, Chía (Colombia).

2 Departamento de Agronomía, Facultad de Agronomía, Universidad Nacional de Colombia, Bogotá (Colombia).

3 Autor para correspondencia. galigarretom@unal.edu.co
} 


\section{ABSTRACT}

Two training systems were evaluated. The trained pea system is an innovation to the traditional systems of training. Vertical training and the new training system is built with new materials have no affect on plant density and allow a better access to machinery. The study encompassed two parts: determination of the appropriate structure for arch training, and evaluation of the economic and technical viability of the proposed system in indeterminate pea compared to the traditional training. The arch training was built with bamboo, polypropylene fiber and meshes. The following variables were evaluated: bamboo distance, hook type between bamboo and size of the mesh. The largest yields were obtained with a pole bamboo distance of two meters, a single hook between bamboo and mesh of $15 \times 17 \mathrm{~cm}^{2}$. In the second part of the trial vertical training showed a lower yield, higher costs of installation and a longer useful life.

Additional key words: planting system, phytosanitory managing, yield, pod quality.

Aprobado para publicación: 01-06-2009

La arveja ha sido cultivada como una fuente importante de alimento humano durante muchos siglos y es reconocida por su gran valor nutricional, ya que representa una alta fuente de proteína y carbohidratos, destacándose como fuente de sacarosa y aminoácidos, incluyendo lisina. También es un alimento de contenidos significativos de minerales y vitaminas, por esta razón ocupa un sitio de preferencia en el ámbito alimenticio de los consumidores de las principales ciudades de Colombia.

FAO (2009) reporta que la arveja en verde se siembra en una basta área geográfica como cultivo comercial; países como China, India, Estados Unidos, Francia, Inglaterra, Egipto y Marruecos se conocen como los mayores productores de arveja comestible durante 2006 y 2007, mientras Warkentin et al. (1995) reportan al este de Canadá los cultivos de arveja con mayor calidad. En Suramérica, Perú es el mayor productor de arveja verde con $86.415 \mathrm{t}$ anuales para 2006 y 86,500 toneladas para 2007, seguido de Colombia con producción de 33.800 t en 2007.
La arveja se produce en 11 de los 32 departamentos de Colombia, pero la mayor producción está concentrada en Cundinamarca y Boyacá, con 35\% y $33 \%$ respectivamente (CCI, 2000). La producción nacional se destina a dos usos diferentes: la verde en vaina para el consumo directo y la seca como semilla, para renovar el ciclo productivo, principalmente por los altos costos que implica su transformación para la obtención del grano seco (SIPSA, 2001).

El cultivo de la arveja en Colombia es considerado rentable, pero implica una inversión inicial para su producción, un alto riesgo de pérdidas por las constantes fluctuaciones de los precios en el mercado, susceptibilidad a problemas fitosanitarios y un alto grado exigencia del consumidor, que rechaza cualquier tipo de daño causado por enfermedades o plagas, representando para el productor un gran reto, que se ve inclinado hacia la utilización de agroquímicos como respuesta efectiva para resolver estas situaciones, pero en la mayoría de los casos el uso inadecuado de químicos es una constante, acarreando consigo graves implicaciones para el productor, el consumidor y el medio ambiente. 
La arveja es la hortaliza de mayor área cosechada en Colombia con 25.658 ha, es decir, el 26,2\% del área hortícola cosechada total en 2004. Sin embargo, su peso en la producción es sustancialmente menor. Esto se reflejó en los bajos rendimientos obtenidos: $1,4 \mathrm{t} \mathrm{ha} \mathrm{h}^{-1}$ en 2000 (CCI, 2000) y 1,24 t ha-1 en 2000 (MADR, 2006).

Existen diversas variedades de arveja como la Piquinegra, la Parda, la Pajarito, la Guatecana y la Bogotana (CCI, 2000), pero el material Santa Isabel ocupa casi la totalidad del área sembrada, ya que satisface los requerimientos del mercado. Esta homogeneidad de la variedad representa una desventaja desde el punto de vista fitosanitario, haciendo que el manejo de enfermedades producidas por patógenos sea cada vez más costosa y difícil (Fenalce, 2002).

En la actualidad no se ofrecen materiales de siembra que satisfagan las necesidades del productor y del mercado y con adaptación a Cundinamarca y Boyacá, como departamentos de mayor área de siembra, ya que según Cousin (1997) son muchos los caracteres que tienen que ser mejorados para satisfacer las necesidades de productores y la industria de alimentos. Una de esas necesidades para los productores es la alta susceptibilidad a las enfermedades que afectan a la planta, siendo las manchas foliares las más limitantes, principalmente el tizón, añublo de la arveja causado por el complejo Ascochyta spp. (Alarcón, 1998), que reduce los rendimientos entre un $20 \%$ y un $50 \%$, demeritando así la calidad de la vaina y los granos cosechados (Tamayo, 2000). Entre los factores que más afectan la incidencia de las enfermedades al cultivo están las altas densidades de siembra, las condiciones medioambientales de alta humedad relativa, la falta de rotación de cultivos y la poca disponibilidad de variedades mejoradas. Aún no se tiene conocimiento sobre la evaluación de los sistemas de tutorado en la incidencia de las enfermedades en arveja.

Las enfermedades causadas por Ascohyta spp. se presentan en todos los lugares del mundo donde se siembra la arveja (Aragona y Porta-Plugia,
1997). Se ha reportado en las regiones templadas de Europa, Norte América, Australia, Nueva Zelanda y en áreas subtropicales de África, Centro y Sur América y Haití (Kraft y Pfleger, 2001), mientras en Colombia, Buriticá (1999) reporta la enfermedad en los departamentos de Cundinamarca, Boyacá y Nariño.

Otra de las enfermedades limitantes del cultivo es el mildeo velloso, causado por el hongo Peronospora viciae f. sp. pisi (Sydow) Boherema \& Verhoeven. la enfermedad ocurre en todas las partes del mundo donde se cultiva la arveja (Kraft y Pfleger, 2001) y en Colombia, Buriticá (1999) la ha reportado en los departamentos de Antioquia, Boyacá, Cundinamarca y Nariño, en Cundinamarca ha llegado a causar perdidas totales de cultivo (Tamayo, 2000). Otras enfermedades reportadas con gran impacto son la antracnosis, causada por Colletotrichum pisi Pat.; el moho gris, causado por Botryotinia fuckeliana (de Bary) Whetzel y la marchitez, causada por Fusarium oxysporum f. sp. pisi (van Hall) Snyd. \& Hans.

De acuerdo con anteriores observaciones, es viable el mejoramiento de algunos de los factores internos de la planta, como lo es la resistencia varietal, y externos para aumentar la productividad hasta alcanzar el máximo genético de la planta. El tutorado es uno de los aspectos externos más importantes que se tiene en cuenta al adoptar un sistema de siembra, ya que está relacionado con el rendimiento y la buena calidad del producto final.

El hábito voluble de la arveja exige un sistema de tutorado que permita la aireación de las plantas y su apropiado manejo, facilitando las labores como aspersiones, control de arvenses, fertilización y cosecha. Para Almanza y Fischer (1993), el tutorado tiene como finalidad cambiar el hábito de crecimiento de la planta, optimizar la distribución de la luz para aumentar la rata fotosintética y mejorar la calidad de los frutos.

Arévalo y Ortega (1995) reportan que con la utilización de tutores en el cultivo de arveja se ob- 
tiene un mayor rendimiento por hectárea y una mejor calidad de frutos. El mayor rendimiento se debe a que los tutores permiten aprovechar mejor el espacio aéreo, disponiendo de mayor área de terreno para sembrar más plantas. La mejor calidad de los frutos se debe a la mayor iluminación que recibe el cultivo, favoreciendo un mejor llenado de las vainas; además, la posición vertical de las plantas contribuye a un control más eficiente de plagas, enfermedades y daños por pájaros.

En Colombia existen diversos sistemas de siembra de arveja en monocultivo, entre los que Castro (1995) destaca los siguientes:

\section{Con tutor vertical}

En las variedades hortícolas de enrame es muy conveniente preparar un dispositivo capaz de soportar los tallos trepadores, ayudando con ello a que éstos se desarrollen y produzcan una mayor cosecha.

Se requieren de 30 a $35 \mathrm{~kg}$ de semilla por ha. Se siembra a distancias entre surcos de 1,0 y $1,2 \mathrm{~m}$, $10 \mathrm{~cm}$ entre sitios, una semilla por sitio o cada $20 \mathrm{~cm}$ dos semillas por sitios, o cada $30 \mathrm{~cm}$ tres semillas por sitio. Para tutorar se usan varas de madera de 2,0 a 2,5 m cada cuatro a cinco $\mathrm{m}$, alambre calibre 16 para colgar allí las plantas en amarre individual con hilaza agrícola cuando la planta tiene de 10 a $20 \mathrm{~cm}$ de altura.

\section{Con tutor horizontal}

Se requieren 30 a $35 \mathrm{~kg}$ de semilla por ha. Se siembra a distancias entre surcos de 1,0 y 1,2 $\mathrm{m}, 10 \mathrm{~cm}$ entre sitios, una semilla por sitio o cada $20 \mathrm{~cm}$ dos semillas por sitio, o cada $30 \mathrm{~cm}$ tres semillas por sitio. Se utilizan varas de 2,5 $\mathrm{m}$ colocadas a una distancia de $4 \mathrm{~m}$, se disminuye la distancia porque no hay utilización de alambre. A medida que va creciendo la planta se van pasando hiladas horizontales cada 15 a 20 $\mathrm{cm}$ durante la etapa de crecimiento (siete a diez hiladas) con polipropileno encanastándola, alternando una por cada lado.

Entre los dos sistemas de tutorado mencionados, el vertical se utiliza en más del $90 \%$ de los cultivos por su facilidad en realizar la labor y por la estabilidad y resistencia del tutor, ya que en el encanaste con frecuencia hay volcamiento de plantas causado por el peso del follaje del cultivo. La desventaja de estos tutorados es que impide la mecanización del cultivo. Una propuesta de innovación en el sistema tradicional es el tutorado en arco sin afectar la densidad de siembra y facilitando el ingreso de maquinaria para el desarrollo de labores durante el ciclo del cultivo.

El sistema de tutorado en arco es una metodología concertada entre los investigadores del proyecto de leguminosas de la Universidad $\mathrm{Na}$ cional de Colombia y profesionales de las entidades Agrointegral Andina S.A. y Syngenta y el agricultor de la Sabana de Bogotá Miguel García, que pretende facilitar el manejo fitosanitario conseguido con el tutor vertical y horizontal al lograr realizar de forma mecanizada las aplicaciones fitosanitarias en zonas donde la mano de obra es escasa, con la opción de tener una optima calidad y sanidad de las vainas, al igual que mayor peso; lograr aumentar la eficiencia fotosintética de la planta; implementar el sistema en la época de invierno; incrementar lar eficiencia en el tiempo de realización de las aplicaciones y tener coberturas homogéneas.

\section{MATERIALES Y MÉTODOS}

El trabajo experimental se llevó a cabo en la finca San Francisco, ubicada en la vereda Laguna Larga, entre las localidades de Madrid y Facatativá (Cundinamarca). El manejo fitosanitario y la fertilización se realizaron de acuerdo con las prácticas corrientes que realiza el agricultor. El material vegetal utilizado fue la variedad Santa Isabel por ser la más cultivada a nivel nacional, y principalmente en la Sabana de Bogotá, ésta 
tiene crecimiento indeterminado y alcanza alturas mayores a $1,60 \mathrm{~m}$. El desarrollo experimental comprendió dos partes. La primera parte fue evaluar la estructura adecuada para realizar el tutorado en arco y la segunda fue el análisis de viabilidad técnica y económica del cultivo de la arveja mediante los dos sistemas de tutorado en estudio: arco y vertical.

El tutorado en arco se instaló a los 60 dds, para ello se usaron guaduas de 1,70 m de longitud y cuatro $\mathrm{cm}$ de ancho. Con estas dimensiones, la guadua, por su flexibilidad, logró formar un arco con $0,4 \mathrm{~m}$ de altura y 1,20 $\mathrm{m}$ de ancho (figura 1a), la distancia entre surcos fue de $1,80 \mathrm{~m}$. El diseño experimental utilizado en esta primera fase fue el de bloques, completamente aleatorio en arreglo factorial con 27 tratamientos y tres repeticio- nes, en las que el factor principal correspondió a la distancia entre guaduas, el factor secundario a enganches entre guaduas y el tercer factor a la referencia de la malla; a continuación se relacionan los diferentes niveles de cada factor:

Distancia entre guaduas: G1: 2 m; G2: 4 m; G3: $6 \mathrm{~m}$. Enganche entre guaduas: E1: un punto; E2: dos puntos; E3: tres puntos. En la figura 1b, se muestra la ubicación de los enganches entre guaduas, se utilizó fibra de $100 \%$ polipropileno referencia $18 \times 5$ monofilamentada, que presentó alta flexibilidad y resistencia. Tipos de malla: M1: malla con $4 \times 4 \mathrm{~cm}^{2}$ de cuadros interiores; M2: malla con $7,5 \times 7,5 \mathrm{~cm}^{2}$ entre cuadros; $\mathrm{M} 3$ : malla con $15 \times 17 \mathrm{~cm}^{2}$ de cuadros interiores; la malla 3 tenía $80 \mathrm{~cm}$ de ancho, las otras dos cubrieron en su totalidad al arco (figura 1c).

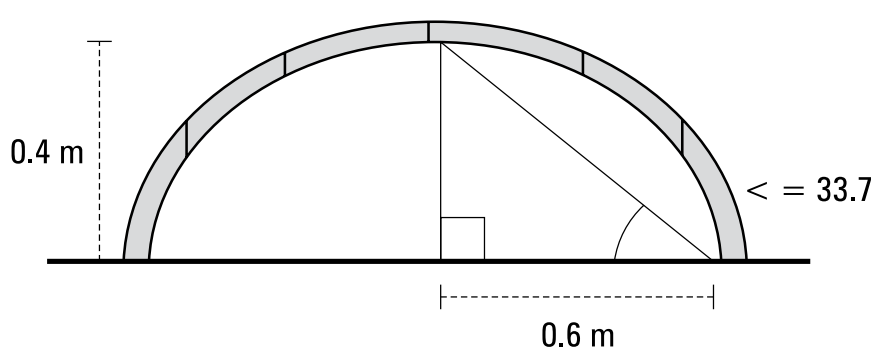

Figura 1a. Estructura del tutorado en arco, utilizando guadua.

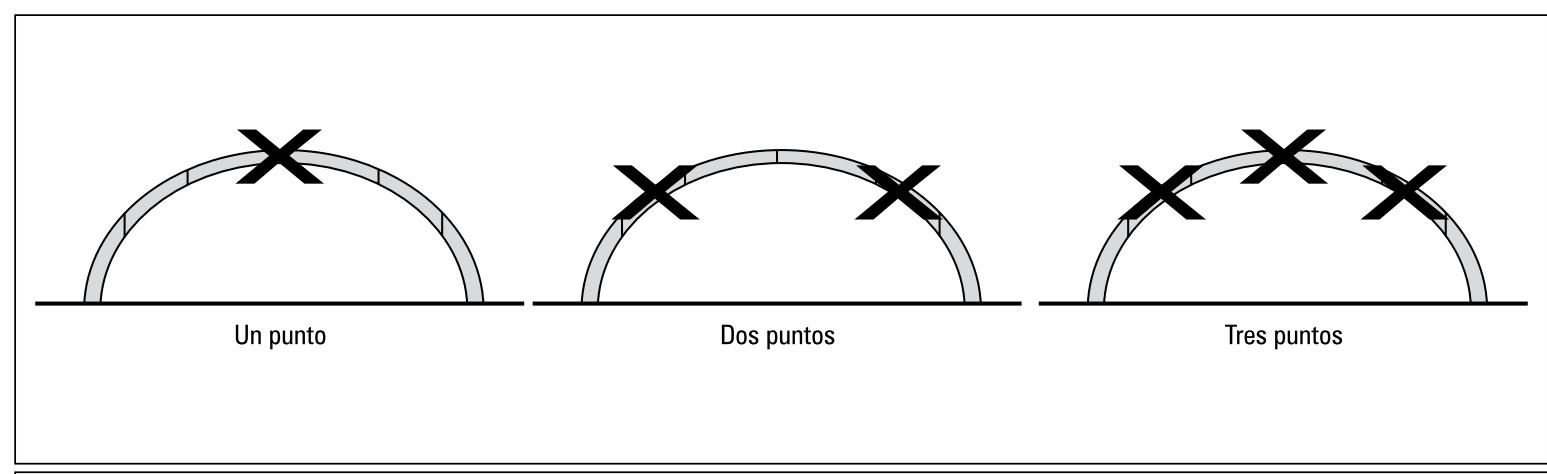

Figura 1b. Posición de los enganches en los arcos de guadua. 


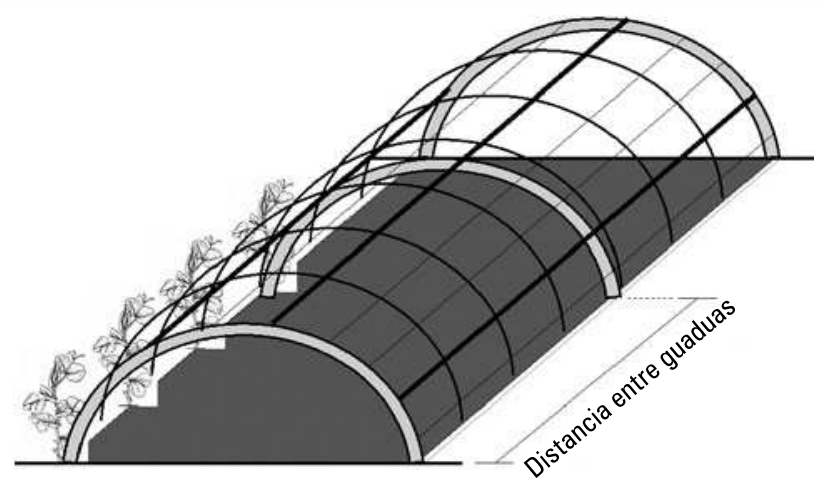

Figura 1c. Estructura del sistema de tutorado en arco

Cada unidad experimental estuvo conformada por $21,6 \mathrm{~m}^{2}$. Las mediciones se realizaron durante todo el ciclo del cultivo, el cual fue de 23 semanas. El rendimiento se obtuvo al cosechar las vainas de cada unidad experimental. Posteriormente, se procedió a determinar los datos de los componentes de rendimiento, los cuales son: número de vainas por planta que se tomó en cinco plantas por tratamiento, numero de granos por vaina evaluado sobre 10 vainas cosechadas, peso de 20 vainas en verde y el peso de 100 granos.

De manera simultánea al experimento de tutorado en arco, se realizó un cultivo de $500 \mathrm{~m}^{2}$ en tutorado vertical. Para el efecto, se utilizaron distancias entre surcos de $1,2 \mathrm{~m}$, varas de 2,5 $\mathrm{m}$ de largo cada $4,6 \mathrm{~m}$, alambre calibre 16 y a los 60 dds se colgaron del tutor las plantas en amarre individual con hilaza agrícola, con recolgado a los 105 y 125 dds. La comparación entre los dos sistemas de tutorado no tuvo un diseño estadístico, se realizó extrapolando las áreas de los dos sistemas de tutorado a una hectárea de producción, para el caso del sistema en arco el tratamiento seleccionado fue distancias entre guaduas de $2 \mathrm{~m}$ y un enganche que, a su vez, fueron los mejores niveles de cada factor evaluado, con un tamaño de hueco de la malla de $15 \mathrm{x}$ $17 \mathrm{~cm}^{2}$ por ser la más económica.

Para cuantificar la enfermedad, se utilizó una escala de severidad basada en la percepción visual. Se establecieron niveles de 1 a 9 , donde 1 es au- sencia de lesiones visibles; $3=2 \%, 5=5 \%, 7=10 \%$ y $9=25 \%$ o más del área de las vainas afectadas, con base en las escalas utilizadas para ascochyta, reportadas por Van Shoonhoven y Pastor-Corrales (1991). La incidencia de la enfermedad se midió como el número de vainas afectadas en cinco plantas por parcela con al menos una cantidad de tejido afectado del $2 \%$, expresando luego estas unidades o vainas como porcentaje de la población total de las unidades cosechadas.

\section{RESULTADOS Y DISCUSIÓN}

\section{Evaluación del sistema de tutorado en arco}

El ciclo del cultivo en las condiciones de la Sábana de Bogotá con el tutorado en arco fue de 21 semanas y se lograron realizar dos pases de cosecha. Del análisis estadístico se reporta en la tabla 1 que hay diferencias significativas en el número de granos por vaina y en el peso de las vainas cuando se varió la distancia entre las guaduas, para este mismo factor las diferencias fueron altamente significativas cuando se cuantificó el número de vainas por planta, también se establecieron diferencias significativas para las variables peso de 100 granos y peso de las vainas en estado verde al utilizar diferente número de enganches entre guaduas, resultados concordantes con lo reportado por Lehman y Lambert, citados por Castro (1995), que concluyeron que, al estudiar el efecto de las distancias de siembra sobre el ren- 
Tabla 1. Resumen del análisis de varianza para los componentes del rendimiento de arveja. Modalidad de tutorado en arco.

\begin{tabular}{|l|c|c|c|c|c|}
\hline \multirow{2}{*}{$\begin{array}{l}\text { Fuente de } \\
\text { variación }\end{array}$} & $\mathrm{GL}$ & $\begin{array}{c}\text { Peso de granos } \\
(100)\end{array}$ & $\begin{array}{c}\text { No. de granos por } \\
\text { vaina }\end{array}$ & $\begin{array}{c}\text { Peso de vainas } \\
\text { verdes (20) }\end{array}$ & $\begin{array}{c}\text { No. vainas verdes } \\
\text { por planta }\end{array}$ \\
\hline G & 2 & 0,40 & $2,65^{*}$ & $34,72^{*}$ & $7,73^{* *}$ \\
\hline$E$ & 2 & $5,20^{*}$ & 0,70 & $18,39^{*}$ & 1,29 \\
\hline$M$ & 2 & 0,70 & 0,61 & 11,62 & 0,60 \\
\hline$G^{*} E$ & 4 & $3,54^{*}$ & 0,96 & 1,18 & 0,96 \\
\hline$G^{*} M$ & 4 & 3,26 & 1,13 & 4,32 & 2,00 \\
\hline$E^{*} M$ & 4 & 0.84 & 0,55 & 1,58 & 3,57 \\
\hline$E^{*} M^{*} G$ & 1,00 & 0,15 & 5,96 & 2,39 \\
\hline \multicolumn{2}{l|}{ Coeficiente de variación (\%) } & 1,83 & 15,47 & 1,61 & 18,47 \\
\hline
\end{tabular}

G, Distancia entre guaduas; $E$, Enganche entre guaduas; $M$, Referencia de malla.

* Diferencias significativas $P \leq 0,05$; ${ }^{*}$ Diferencia altamente significativa $P \leq 0,01$.

dimiento y sus componentes en diferentes leguminosas de grano, el componente más afectado es el número de vainas por planta $\mathrm{y}$, en menor grado, el número de granos por vaina y el peso de los granos.

La distancia entre guaduas de dos metros generó mayor rendimiento en vainas por planta y afectó favorablemente a las variables número de granos por vaina y peso de las vainas, también asociadas con el rendimiento. El comportamiento se dio porque las plantas estuvieron a mayor altura del suelo, respecto a las otras distancias de cuatro y seis metros entre guaduas, originando mayor aireación entre las plantas, lo cual favorece el desarrollo fisiológico y reproductivo. Al haber mayor fotosíntesis y translocación de asimilados a las estructuras reproductivas, también hay menor afectación por enfermedades, lo cual se refleja de manera directa en el mayor peso de los granos y en el peso de las vainas (tabla 2).

En cuanto a la estructura del tutorado, es importante considerar que con la fibra de polipropileno, que tiene una fuerza de tensión de $79 \mathrm{~kg}$ (Ciplas, 2005) y se sostiene mediante los arcos de guadua, a medida que es mayor la distancia entre guaduas, la fibra realiza mayor fuerza de tensión, lo que provocó que a las distancias mayores a $2 \mathrm{~m}$ no cumpliera su objetivo de forma satisfactoria y se cediera, permitiendo que las vainas rozaran con el suelo.

Para la fuente de variación enganche entre guaduas se observaron diferencias significativas en el enganche entre un punto y dos puntos, mientras que entre el enganche a un punto $y$ tres puntos las diferencias fueron altamente significativas.

Tabla 2. Resultados de los componentes de rendimiento de la arveja en el tutorado en arco variando la distancia entre guaduas.

\begin{tabular}{|c|c|c|c|}
\hline Distancia entre guaduas (G) & No. de vainas por planta & Peso de vainas verde (20) & No. de granos por vaina \\
\hline $2 \mathrm{~m}$ & $7,04 \mathrm{a}$ & $135,34 \mathrm{a}$ & $4,84 \mathrm{a}$ \\
\hline $4 \mathrm{~m}$ & $6,18 \mathrm{~b}$ & $134,81 \mathrm{a}$ & $4,68 \mathrm{ab}$ \\
\hline $6 \mathrm{~m}$ & $5,74 \mathrm{c}$ & $133,42 \mathrm{~b}$ & $4,60 \mathrm{~b}$ \\
\hline
\end{tabular}

Promedios con letras distintas indican diferencia significativa según la prueba de Duncan $(P \leq 0,05)$. 
La media más alta se presentó en el enganche entre guaduas a un punto (tabla 3 ), tal vez porque la planta tiende a permanecer más erecta que en dos y tres enganches, lo que favorece la traslocación de asimilados hacia las estructuras reproductivas. La referencia de la malla no presentó diferencias significativas en ninguno de los componentes del rendimiento, por esta razón la más conveniente fue la referencia $15 \times 17 \mathrm{~cm}^{2}$ de cuadros interiores, ya que fue la de menor costo.

El tutorado en arco, por ser un trabajo experimental inicial, no posee un paquete tecnológico de cultivo. Muchas de las labores realizadas fueron basadas en prácticas que se realizan con el tutorado vertical y otras prácticas se implementaron con el fin de dar solución a limitantes de la producción.

\section{Análisis técnico y económico de los sistemas de tutorado vertical y en arco}

Durante el ciclo del cultivo no se presentaron diferencias en el manejo de plagas con los sistemas de tutorado, en los primeros estados del cultivo se hizo necesario el control químico de trozadores del tallo (Spodoptera sp. y Agrotis ipsilon), mientras para el resto del ciclo no se presentó daño por insectos plaga.

El manejo de malezas fue una de las labores dispendiosa de realizar bajo el tutorado en arco, debido a que los $40 \mathrm{~cm}$ de altura que posee el arco dificultan el acceso del personal para realizar la labor manual, mientras que en el tutorado tradicional la labor se realiza de manera fácil y con mayor rapidez. Una vez instalado el sistema de tutora- do en arco, se requiere el doble de jornales para la realización de la labor de control de las malezas respecto al sistema de tutorado vertical.

Las enfermedades con mayor incidencia y severidad fueron el mildeo velloso y el tizón de la arveja o pecoseo, y presentaron diferencias en cada sistema de tutorado. El mildeo velloso, causado por Peronospora viciae f. sp. pisi (Sydow) Boherema \& Verhoeven, presentó mayor incidencia bajo el tutorado vertical, mientras que el tizón o "pecoseo" de la arveja, causado por el complejo Ascochyta pisi, Micosphaerella pinodes y Phoma medicaginis (micosphaerella medicaginis)(Kraft y Pfleger, 2001), presentó la mayor incidencia en el tutorado en arco, comportamiento que muestra una de las diferencias a resaltar entre los dos sistemas (figuras 2 y 3 ).

El mildeo velloso recubre el envés de las hojas de un micelio grisáceo o gris-marrón, en el haz, la infección se manifiesta con manchas de color amarillo-verdoso, también infecciones sistémicas provocan clorosis, deformación de los brotes y enanismo. Ocurre en condiciones frías $\left(15^{\circ} \mathrm{C}\right)$, el bajo número de horas luz en días consecutivos es un factor determinante para el desarrollo de la enfermedad, la humedad ambiental y el mantenimiento de una película de agua en la superficie de las hojas favorece la germinación de oosporas y desarrollo de los esporangios con la consiguiente propagación de la enfermedad (Kraft y Pfleger, 2001).

Para el tutorado vertical, la enfermedad mildeo velloso alcanzó los mayores valores de incidencia en las vainas en los tres pases de cosecha con valores de $8 \%, 21 \%$ y $30 \%$ de vainas afectadas respectivamente en primero, segundo y tercer pase,

Tabla 3. Resultados de los componentes de rendimiento en arveja tutorada en arco variando el número de enganches entre guaduas.

\begin{tabular}{|l|c|c|}
\hline \multicolumn{2}{|c|}{ Enganche entre guaduas (E) } & Peso de granos (100) \\
\hline Un punto & $52,01 \mathrm{a}$ & $135,25 \mathrm{a}$ \\
\hline Dos puntos & $51,40 \mathrm{~b}$ & $134,48 \mathrm{ab}$ \\
\hline Tres puntos & $51,30 \mathrm{~b}$ & $133,81 \mathrm{~b}$ \\
\hline
\end{tabular}

Promedios con letras distintas indican diferencia significativa según la prueba de Duncan $(P \leq 0,05)$. 


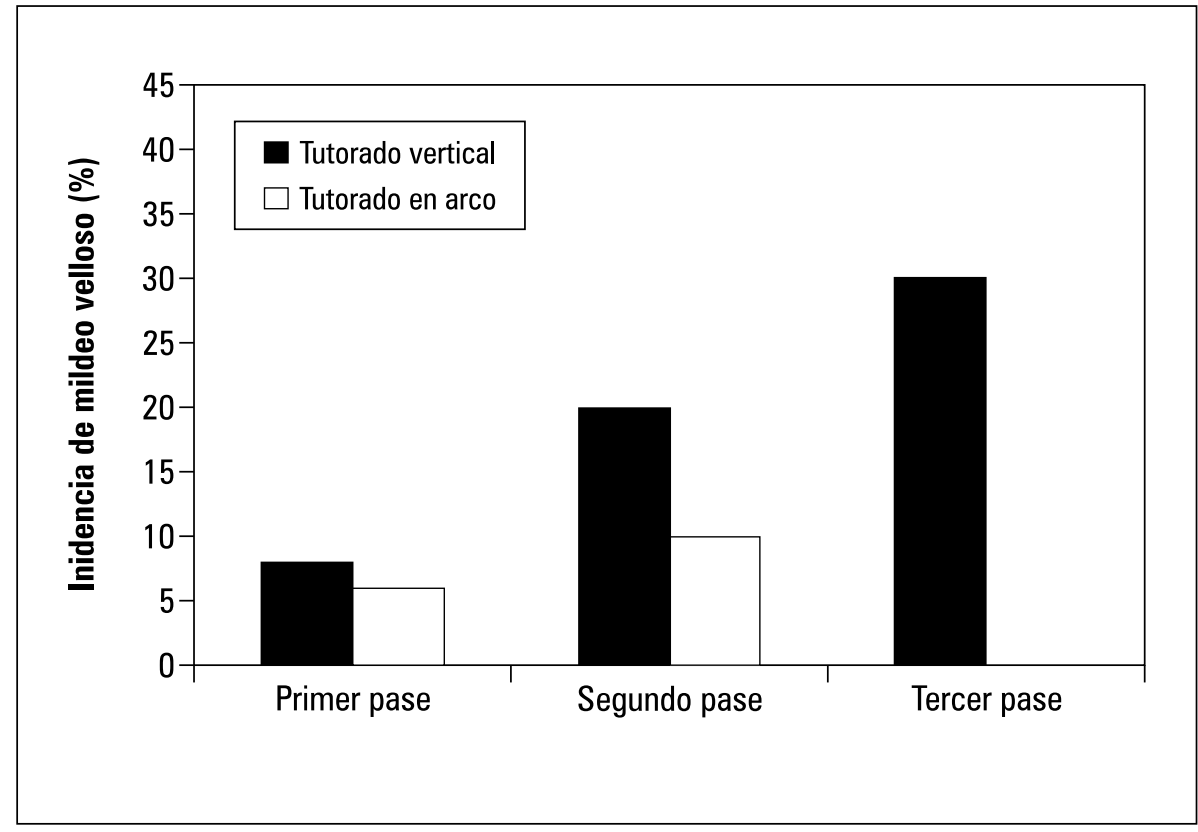

Figura 2. Incidencia de mildeo velloso sobre vainas cosechadas.

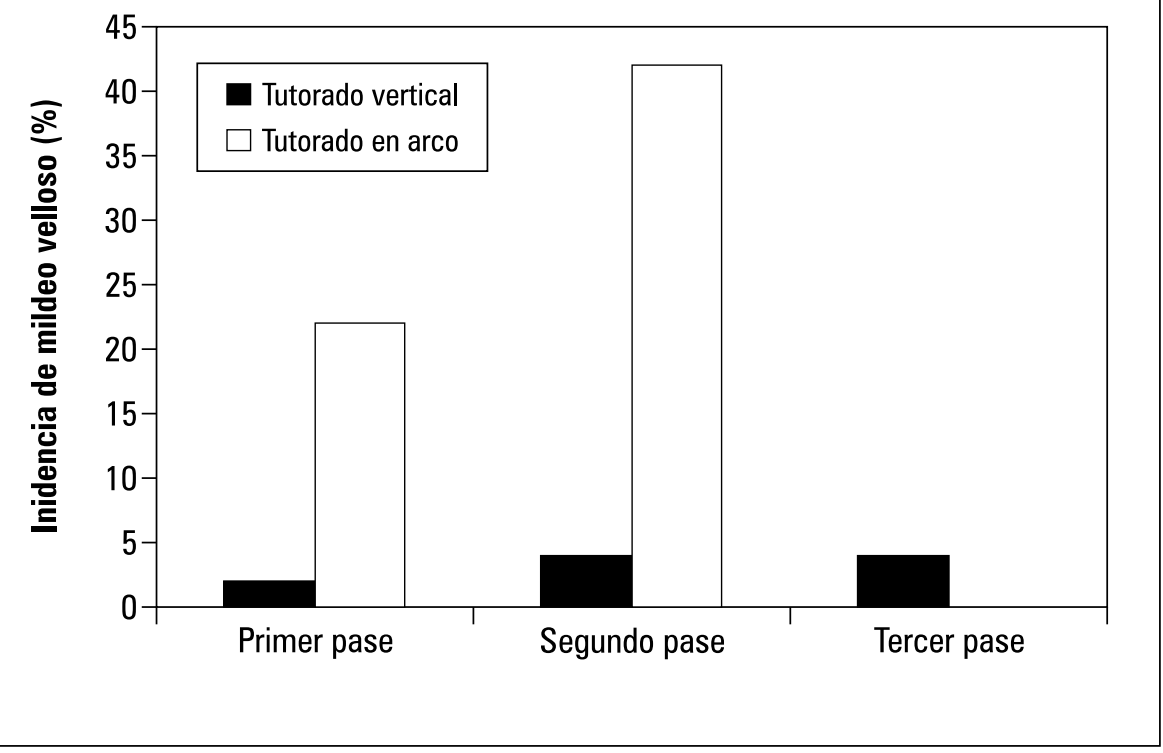

Figura 3. Incidencia del tizón o pecoseo de la arveja sobre vainas cosechadas. 
factor que tiene alto impacto en la reducción de la productividad del cultivo de arveja (figura 2). Por el contrario, en el sistema en arco la incidencia del mildeo velloso fue baja con promedio de porcentaje del $6 \%$ y $10 \%$ en primero y segundo pase respectivamente, la variación de estos resultados pudo ser causada porque la densidad de las plantas fue mayor en el tutor vertical y se presenta sombreamiento de las plantas de un surco con las de los surcos vecinos, principalmente en los dos primeros tercios de la planta, también porque hay alta humedad ambiental favorable al establecimiento y daño del patógeno, mientras en el sistema de arco no hay sombreamiento entre plantas de un surco con los surcos vecinos, aun cuando las plantas en su tercio inferior que da sobre la malla también presentan alta humedad y tienen poca aireación.

El pecoseo prevalece en condiciones de lluvia y alta humedad relativa, los cultivos sin tutor $y$ con deficiente control de las malezas son más susceptibles. Los síntomas y signos se hacen más evidentes después de floración, en el tercio inferior de la planta. En las hojas se forman lesiones circulares de 2 a $10 \mathrm{~mm}$ de color café. En las vainas se forman lesiones deprimidas que dañan la presentación y pueden llegar a causar daño en la semilla (Tamayo, 2000).

Contrario al caso anterior, el pecoseo fue limitante en la producción bajo el tutorado en arco, lo cual disminuyó la cantidad de vainas verdes de óptima calidad para su comercialización. La incidencia de la enfermedad sobre las vainas cosechadas en el cultivo con tutor vertical fue inferior al $5 \%$, permitiendo obtener una cosecha de muy buena calidad por este factor, en el sistema en arco se alcanzo el $22 \%$ y $42 \%$ de vainas con daño de tizón en primer y segundo pase respectivamente, deteriorando drásticamente el producto para la venta (figura 3). La alta incidencia del daño fue causada porque en el costado de las plantas que cae sobre el tutor no recibe aplicaciones de control químico para la enfermedad, excepto lo que la planta logre trasportar a esos tejidos, lo cual, unido a factores de alta población de las malezas y alta densidad del follaje de la planta, origina la humedad ambiental favorable al establecimiento y desarrollo del patógeno, afectando drásticamente la producción y calidad del producto. Factores que tuvieron mejor control en el sistema de tutorado vertical. La incidencia de ambas enfermedades aumentó en cada pase de cosecha, debido a que no se realizaron aplicaciones fitosanitarias a partir de $130 \mathrm{dds}$.

El ciclo del cultivo en las condiciones de la Sábana de Bogotá con el tutorado vertical fue de 23 semanas y se logró realizar tres pases de cosecha, uno más que al tutorado en arco, aunque este último sólo representó el 9\% del rendimiento total en el tutorado vertical. Las diferencias grandes de producción entre los dos sistemas se presentaron en el segundo pase, el cual representó el 58\% de la producción total en el tutorado vertical y el $62 \%$ de la misma en el tutorado en arco. E1 rendimiento del tutorado vertical superó en un $36 \%$ al tutorado en arco (figura 4). Por tanto, la propuesta más viable para la Sabana de Bogotá es el sistema de tutorado vertical, ya que con él se obtienen rendimientos de $9,7 \mathrm{t} \mathrm{ha}^{-1}$ con tres pases de cosecha, mientras que bajo el sistema de tutorado en arco los rendimientos son de 6.2 ton ha con dos pases de cosecha (figura 4).

El mayor rendimiento del cultivo en el sistema de tutorado vertical se debe a dos aspectos. El primer aspecto a considerar es el programa tecnológico que se ha desarrollado para las condiciones de la Sabana de Bogotá, el cual consiste en la adecuada realización de la preparación del terreno, fertilización, control de malezas y aspersiones para el control de enfermedades e insectos plagas del cultivo, proveniente de varios años de experiencia y ensayos realizados por los agricultores, los investigadores y los profesionales que asisten a los cultivos. El segundo aspecto es la eficiencia fotosintética de la planta en el tutorado vertical, al lograr las plantas recibir mayor iluminación 


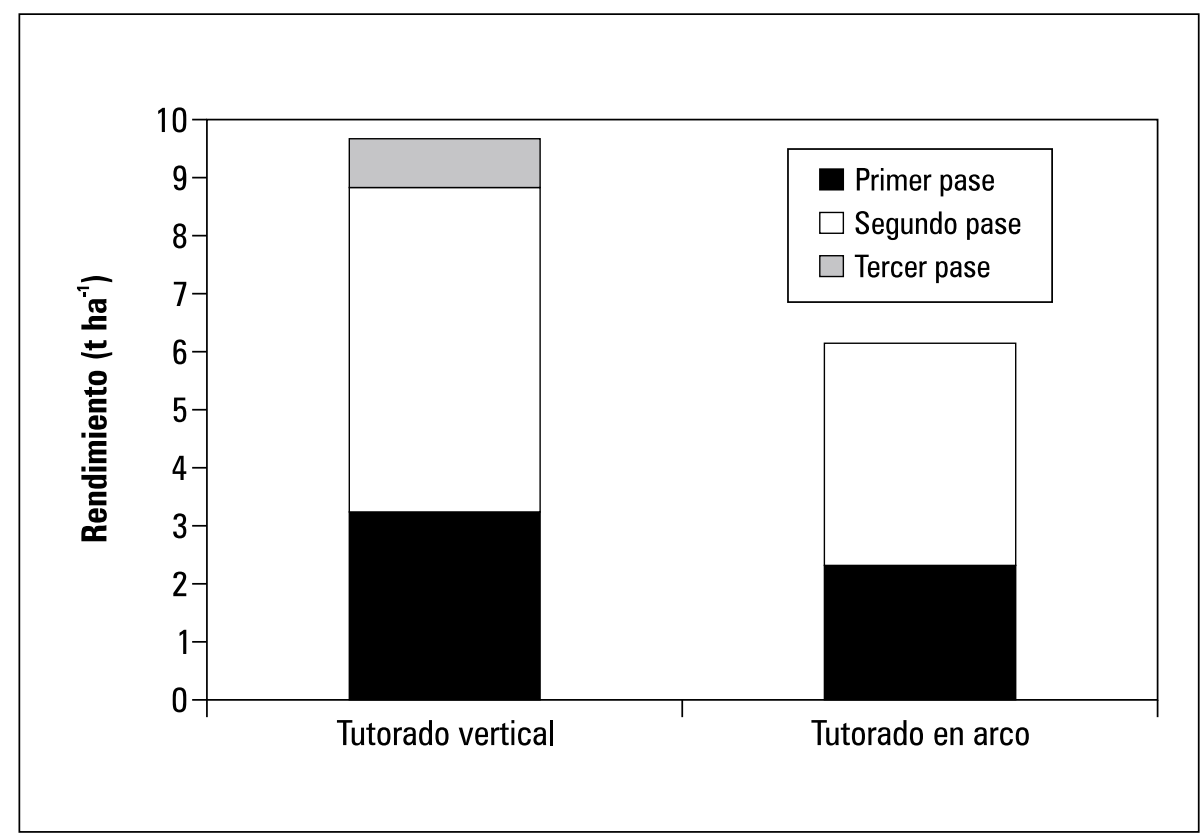

Figura 4. Rendimiento del tutorado vertical vs. el tutorado en arco en condiciones de la Sabana de Bogotá.

en todo su canopy y aprovechar mejor el espacio aéreo, favoreciendo una mejor distribución de las vainas a lo largo del tallo principal y las ramas laterales, un mejor llenado de las vainas y una vainas con menor daño por Ascochyta como la enfermedad que más limita la producción del cultivo.

La cosecha fue la labor más limitante del tutorado en arco, porque hay que levantar las plantas para recoger las vainas que se han enredado en la malla, actividad dispendiosa porque las plantas son de hábito indeterminado y se conectan y amarran por zarcillos, lo cual dificulta la labor $y$, en consecuencia, se empleó más tiempo que la cosecha en el tutorado vertical. En el tutorado vertical, el operario realiza la labor de cosecha de pie y sin agacharse, mientras que en el tutorado en arco la labor se tenía que comenzar a hacer a una altura de $40 \mathrm{~cm}$ del piso, lo que no permitió que rindiera por las constantes inclinadas de los recolectores. En el primer pase de cosecha con el tutorado en arco las plantas fueron maltratadas por intentar recoger las vainas que se encontra- ban ocultas, en el segundo pase de cosecha también se presentó esta situación, pero los recolectores manejaron con más cuidado la labor.

Los materiales que se emplearon para el tutorado en arco se encuentran en la capacidad de cumplir más de cuatro ciclos de cultivo, puesto que la guadua es de alta resistencia, mientras que el tutorado vertical conformado por varas de eucalipto es reutilizado para máximo tres cosechas, lapso de tiempo en el cual se descompone.

Al considerar los costos de instalación del tutorado vertical en $100 \%$ y compararlo con el tutorado en arco, que superó en $151 \%$ los costos del tutorado vertical, resulta una opción no viable para los productores (tablas 4 y 5 ). La mano de obra es uno de los ítems en los que se descompensa el tutorado en arco, ya que se emplea mayor número de jornales y ante todo mayor tiempo para la instalación. La cantidad de semilla que se emplea en el tutorado vertical es mayor (tabla 4) que la que se emplea bajo el tutorado en arco (tabla 5) por las distancias de siembra entre los surcos. 
Tabla 4. Costos de instalación (en pesos colombianos) del cultivo de arveja bajo el tutorado vertical por hectárea (año 2006).

\begin{tabular}{|l|l|r|r|r|}
\hline \multicolumn{1}{|c|}{ Insumo } & \multicolumn{1}{|c|}{ Unidad } & Cantidad & Valor unitario & Valor total \\
\hline Semilla & $\mathrm{kg}$ & 28 & 6.000 & 168.000 \\
\hline Varas & Unidad & 1450 & 430 & 623.500 \\
\hline Alambre & $\mathrm{kg}$ & 250 & 2.800 & 700.000 \\
\hline Hilaza & Cono & 38 & 8.000 & 304.000 \\
\hline Mano de obra & Jornal & 32 & 30.000 & 960.000 \\
\hline Total & & & & 2.755 .500 \\
\hline
\end{tabular}

Tabla 5. Costos de instalación (en pesos colombianos) del cultivo de arveja bajo el tutorado en arco por hectárea (año 2006).

\begin{tabular}{|l|l|r|r|r|}
\hline \multicolumn{1}{|c|}{ Insumo } & Unidad & Cantidad & Valor unitario & Valor total \\
\hline Semilla & $\mathrm{kg}$ & 3 & 6.000 & 18.000 \\
\hline Guadua & Unidad & 2750 & 1.200 & 3.300 .000 \\
\hline Fibra & Rollo & 3 & 43.639 & 130.917 \\
\hline Malla & $\mathrm{m}$ & 5560 & 400 & 2.224 .000 \\
\hline Mano de obra & Jornal & 42 & 30.000 & 1.260 .000 \\
\hline Total & & & 6.932 .917 \\
\hline
\end{tabular}

\section{CONCLUSIONES}

Las mayores limitantes bajo el sistema de tutorado en arco fueron el control de malezas luego de haber instalado el tutorado y la labor de cosecha, lo que aumentó la cantidad de mano de obra para realizar estas actividades con respecto al tutorado tradicional.

La propuesta de tutorado en arco puede llevarse a cabo en Colombia, pero para ello se necesitaría innovar más en su estructura, para llegar a los rendimientos que se están obteniendo con el sistema de tutorado vertical en la Sabana de Bo- gotá, ya que ella representa una alternativa que puede reducir los costos de manejo en el sistema de producción al permitir el acceso del tractor en el cultivo para aplicaciones fitosanitarias.

Las investigación se debe encaminar a mejorar el sistema de tutorado vertical mediante la investigación de materiales de siembra con mayor rendimiento para la Sabana de Bogotá, el perfeccionamiento de las labores de instalación del tutorado y con el correcto uso de los agroinsumos para el manejo de las enfermedades e insectos plagas. 


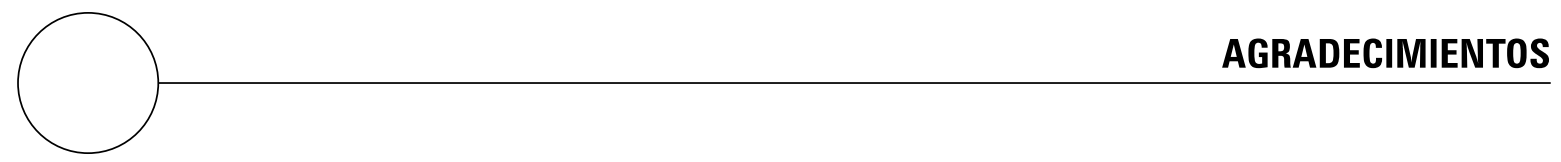

Los autores expresan agradecimientos a los ingenieros Rodrigo Prada y Emilio García por la colaboración y aportes en la ejecución de este trabajo; a Miguel García, por generar la idea del sistema de tutorado en arco con el propósito de dar soluciones prácticas a las problemáticas fitosanitarias del cultivo de arveja y por la colaboración en la elaboración de este trabajo; a Agrointegral Andina S.A. y Syngenta, entidades interesadas en estimular la innovación en los sistemas de producción y por su aporte financiero para la realización de la investigación; al ingeniero José Manuel García y al administrador Néstor Hernández por sus sugerencias técnicas en el sistema de producción de arveja.

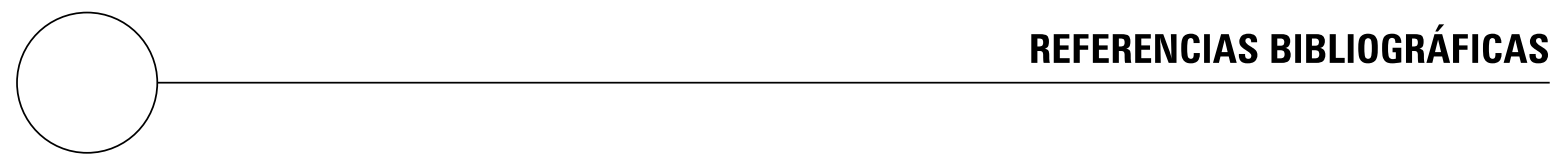

Alarcón, P. 1998. Incidencia y severidad del tizón o añublo (Ascochyta spp.) en dos variedades de arveja ( $P$ isum sativum var. Arvense (L.) Poir) con fertilización convencional y biofertilizantes en el municipio de Pasca (Cundinamarca).Tesis de maestría en Ciencias Agrarias. Facultad de Agronomía, Universidad Nacional de Colombia, Bogotá.

Almanza, P. y G. Fischer. 1993. Nuevas tecnologías en el cultivo de la uchuva. Agro-Desarrollo 4(1-2), 292304.

Aragona, M. y A. Porta-Plugia. 1997. Improvement of grins y legumes. General part: Diseases. Field Crops Res. 53(1), 17-30.

Arévalo, C. y V. Ortega. 1995. Uso de tutores en el cultivo de la arveja. Instituto Nacional de Investigación Agraria, Perú.

Buriticá, P. 1999. Directorio de patógenos y enfermedades de las plantas de importancia económica en Colombia. Produmedios, Bogotá.

Castro, M. 1995. Evaluación de arveja voluble (Pisum sativum L.) en diferentes densidades y sistemas de siembra en la región de Simijaca (Cundinamarca). Trabajo de grado. Facultad de Agronomía, Universidad Nacional de Colombia, Bogotá.

Ciplas S.A. 2005. Our products. En: http://www.ciplas. com/; consulta: enero de 2009.

Corporación Colombia Internacional (CCI). 2000. Manual del exportador de fritas, hortalizas y tubérculos en Colombia. En: http://www.cci.org.co/ Manual\%20Exportador/desempeno_prod/princ prod_mapas/princ_prod11.htm; consulta: diciembre de 2008.

Cousin, R. 1997. Peas (Pisum sativum L.). Field Crops Res. 53, 111-130.

FAO. 2009. Faostat. Organización de las Naciones Unidas para la agricultura y la alimentación. En: http:// faostat.fao.org/; consulta: 23 de abril de 2009.

Federación Nacional de Cultivadores de Cereales y Leguminosas (Fenalce). 2002. Conocimiento Técnico: Amarillamiento en arveja. En: http://www. fenalceorg.c_Cuotas.htm; consulta: diciembre de 2008. 
Kraft, J. y F. Pfleger. 2001. Compendium of pea diseases. APS Press, St. Paul, MN.

Ministerio de Agricultura y Desarrollo Rural (MADR). 2006. Acuerdo de competitividad de la cadena de hortalizas. Observatorio agrocadenas Colombia. 19 p. En: http://www.agrocadenas.gov.co/home.htm; consulta: diciembre de 2008.

SIPSA. 2001. Relaciones de precios y consumo de las arvejas seca y verde en vaina. Boletín mensual No. 43. Agosto-Septiembre de 2001. En: http//:www. cci.org.co/publicaciones/Sipsa/sipsa43.doc; consulta: diciembre de 2008.
Tamayo, P. J. 2000. Enfermedades del cultivo de la arveja en Colombia: Guía de reconocimiento y control. Boletín técnico, Corpoica. Produmedios, Bogotá.

Van Shoonhoven, A. y M. Pastor-Corrales (eds.). 1991. Sistema estándar para la evaluación de germoplasma de fríjol. Centro Internacional de Agricultura Tropical (CIAT), Cali, Colombia.

Warkentin, T.D.; K.Y. Rashid y A. Xue. 1995. Fungicidal control de ascochyta blight of field pea. Can. J. Plant Sci. 75, 67-71. 